Open Access

\title{
A dynamical model for heart remodeling during the two phases of pulmonary arterial hypertension
}

\author{
Adrien Kerfourn ${ }^{1,2+*}$, Bouchra Lamia ${ }^{3}$, Jean-François Muir ${ }^{3}$ and Christophe Letellier ${ }^{1,2+}$
}

\author{
${ }^{*}$ Correspondence: \\ adrien.kerfourn@coria.fr \\ †Equal Contributors \\ ${ }^{1}$ CORIA UMR 6614 - Normandie \\ University, CRNS-Université et INSA \\ de Rouen, Campus Universitaire du \\ Madrillet, F-76800 Saint-Etienne du \\ Rouvray, France \\ ${ }^{2}$ ADIR Association, Avenue du \\ Maréchal Juin, 76031 Rouen cedex, \\ France \\ Full list of author information is \\ available at the end of the article
}

\begin{abstract}
Background: Pulmonary arterial hypertension is a rare and lethal disease affecting small diameter pulmonary arteries and leading to a progressive increase of the right vascular resistances. Patients with such a disease have no specific symptom, a feature which delays the diagnosis by 18 months to 2 years in average. In most cases, pulmonary arterial hypertension is diagnosed when the cardiac output is already reduced, inevitably leading to death when the disease is not efficiently treated. During the evolution of the disease, the right ventricle is clearly affected in two different ways: first the thickness of its walls increases (compensation) and second the ventricle inflates (decompensation). The latter phase remained unexplained.
\end{abstract}

Methods: We developed a dynamical model of the cardiovascular system in order to explain such a feature by regulation mechanisms. Since pulmonary arterial hypertension is a slowly evolving pathology, we took into account long-term regulation mechanisms as the myocardial development (muscular heart development); we only considered the heart rate variations among the short-term regulation mechanisms.

Results: Using a static model, we showed that the two phases observed during the evolution of pulmonary arterial hypertension can be explained by the interplay between the right and left ventricles. We then showed that our dynamical model can reproduce the heart remodeling during these two phases (compensation and decompensation of the right ventricle). After the compensation phase, the right ventricle can no longer maintain the cardiac output without the help of the left ventricle, which produces a part of the required work with the side effect of inflating the right ventricle.

Conclusion: By taking into account slow regulation mechanisms, the cardiac remodeling during pulmonary arterial hypertension was reproduced. We thus showed that the two phases observed during the increase in the pulmonary arterial resistance result from an interplay between the left and right ventricles.

Keywords: Pulmonary arterial hypertension, Heart remodeling, Right ventricle dysfunction, Nonlinear dynamical model

\section{照 Springer}

(c) 2016 Kerfourn et al. Open Access This article is distributed under the terms of the Creative Commons Attribution 4.0 International License (http://creativecommons.org/licenses/by/4.0/), which permits unrestricted use, distribution, and reproduction in any medium, provided you give appropriate credit to the original author(s) and the source, provide a link to the Creative Commons license, and indicate if changes were made. 


\section{Background}

Pulmonary arterial hypertension is a rare and lethal disease which can designate right ventricular dysfunction, mitral valve stenosis, congenital heart disease, pulmonary parenchymal disease, interstitial lung disease, etc. [1]. This pathology affects small diameter pulmonary arteries and leads to a progressive increase in the right vascular resistances [2]. It was first identified by ERNST VON ROMBERG in 1891 [3]. The hemodynamic definition of a pulmonary arterial hypertension is that the mean pulmonary arterial pressure at rest greater than $25 \mathrm{mmHg}[2,4]$. This disease can be hereditary or can occur in a sporadic way [2].

Patients with a pulmonary arterial hypertension have no specific symptom and commonly present fatigue and dyspnea [4], a feature which delays on average the diagnosis by 18 months to 2 years [1]. During the first phase of the disease, the right ventricle compensates the increase of the pulmonary resistance, in such a way that i) the cardiac output is preserved and ii) the mean hemodynamic is slightly or even not affected [5]. A second phase occurs when the right ventricle can no longer ensure the cardiac output due to the increased pulmonary resistance; it thus decompensates and inflates, the latter feature being not yet explained. In most cases, pulmonary arterial hypertension is diagnosed only when the cardiac output is already reduced [4], affecting the survival prognosis when the disease is not efficiently treated [1, 4]. Thus, patients can suffer from idiopathic pulmonary hypertension during 2.8 years on average before receiving specific treatments [2].

The right ventricle remodeling is concomitant with an increase in the pulmonary resistance (Fig. 1). When the pulmonary arterial pressure progressively increases from a normal value (Fig. 1a) to a value corresponding to a light pulmonary hypertension (Fig. 1b), pulmonary arteries are slightly vasoconstricted, a feature which can be associated with an early loss of endothelial cells; during this phase the right ventricle becomes hypertrophied [5]. When the pulmonary arterial hypertension further increases due to a vascular remodeling (Fig. 1c), the right ventricle can no longer compensate the rise of the pulmonary vascular resistance, inducing a failure. The right ventricle thus inflates. This phase is also associated with a proliferation of endothelial cells.

In the present work, our objective is to show that, when a pulmonary arterial hypertension develops, the two phases observed in the right ventricle can be understood when hemodynamical interactions between the right and left sides of the heart are taken into account (the mechanical coupling between the two sides of the heart via the septum is here neglected). Since the right ventricle can no longer correctly provide the required cardiac output, we assumed that the left ventricle greatly assists the right ventricle, thus inducing an increase of the pressure in the venous systemic circuit (therefore in the right atrium input), which in turns produces an inflation of the right ventricle.

The first modeling of the cardiovascular system dates back from the 19th century: it was used to understand how the cardiovascular system works in physiological and pathological cases [6]. In 1735, HALES measured for the first time the blood pressure of the arterial system and noticed that it changes during the cardiac cycle [7]. He also suggested that this pressure variation was the consequence of the arterial elasticity. In 1827 WEBER [8] proposed a comparison between the arterial elasticity and the Windkessel effect observed in a pumping system of fire service [9]. Such analogy then allowed to explain the exponential fall of the aortic pressure during the diastole with a characteristic time $\tau=R_{S} C_{\text {aorta }}$ 


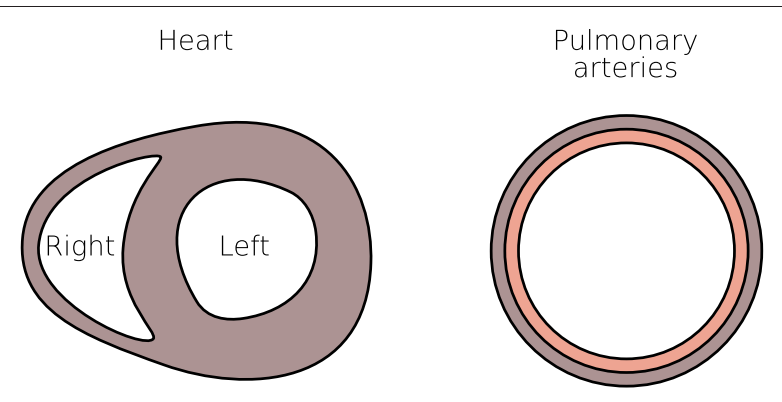

(a) Physiological configuration
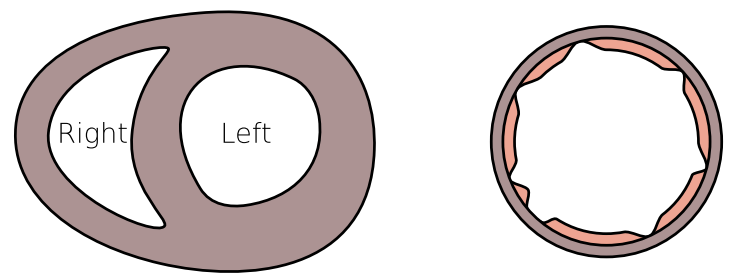

(b) Compensated state of the right ventricle
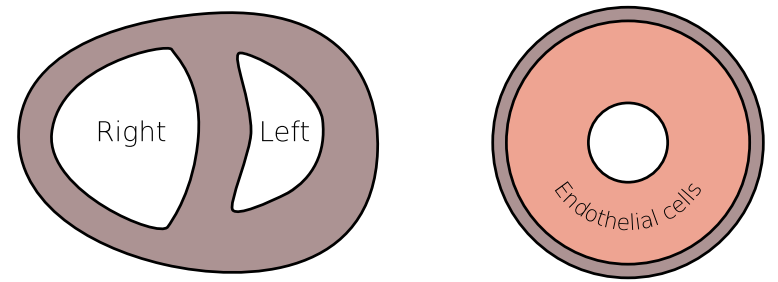

(c) Failure state of the right ventricle

Fig. 1 Schematic progression of a pulmonary vascular disease and its effects on the heart and pulmonary arteries geometry. Starting from a physiological situation (a), the pulmonary arterial pressure slightly increases, inducing an hypertrophy of the right ventricle and a slight vasoconstriction of pulmonary arteries associated with an early loss of endothelial cells: this is the compensation phase $(\mathbf{b})$. A significant increase of the pulmonary arterial pressure due to a strong diameter reduction of pulmonary arteries induces a serious inflation of the right ventricle associated with an endothelial cells proliferation: this is the failure (decompensation) phase (c). Adapted from [5]

where $R_{S}$ is the systemic resistance and $C_{\text {aorta }}$ the aortic compliance. Other models inherited from this approach were thereafter proposed and took into account the blood inertia through an inductive effect $[9,10]$.

The weakness of these models is that they did not describe the cardiovascular system as a whole. In 1964, DE PATER and VAN DEN BERG suggested an electrical analogy of the different hemodynamic components to develop a model of the entire circulatory system which was validated by numerical simulations [11]. In this model, the cardiovascular system was split in two parts: the heart as an active component and the blood vessels as passive components.

Many recent models of the cardiovascular system used a similar structure. Depending on the specific pathology they were supposed to explain, these models were made of some components which were only described simply while some others were more accurately detailed [12-14]. Among dynamical models for the whole cardiovascular system, only a few of them took into account the cardiac output regulation [15]. When pulmonary arterial hypertension is considered, a description of fast (as heart rate variations) and slow (as myocardial development) regulation mechanisms is required to 
understand the two phases observed during the development of this disease. In the 1960s and 1970s, GUYTON et al. proposed some descriptions of the different regulation mechanisms [16, 17], but their model became rather complex and was subject to some criticisms [18].

To validate our hypothesis according which the left ventricle must assist the right one to preserve the cardiac output, we started with a simple static model to compare the power delivered by each ventricle in different situations. We then developed a dynamical model of the cardiovascular system to better understand how the regulation mechanisms induce a heart remodeling due to chronic disease and to reproduce the different phases occurring during a pulmonary arterial hypertension as previously discussed (Fig. 1). This will be achieved by modeling the cardiovascular system of a subject initially healthy and whose pulmonary resistance is slowly increased.

\section{Methods}

\section{A simple static model}

We started with a simple static model: all capacitive and inductive effects were neglected. Thus we described the left side of the heart (made of the left atrium and the left ventricle) and the right side of the heart (the right atrium and the right ventricle) by voltage generators; the systemic and pulmonary circuits were only considered as resistive elements (Fig. 2). We also supposed that the pulmonary venous pressure remains constant.

\section{Normal situation}

Let us assume that the cardiac output $Q_{c}$ of a healthy subject at rest is $51 /$ min. Mean pressures at different locations in the cardiovascular system are

$$
\left\{\begin{array}{lr}
P_{\mathrm{aa}}=95 \mathrm{mmHg} & \text { aorta } \\
P_{\mathrm{vc}}=3 \mathrm{mmHg} & \text { vena cava } \\
P_{\mathrm{pa}}=11.6 \mathrm{mmHg} & \text { pulmonary artery } \\
P_{\mathrm{pv}}=8 \mathrm{mmHg} & \text { pulmonary vein. }
\end{array}\right.
$$

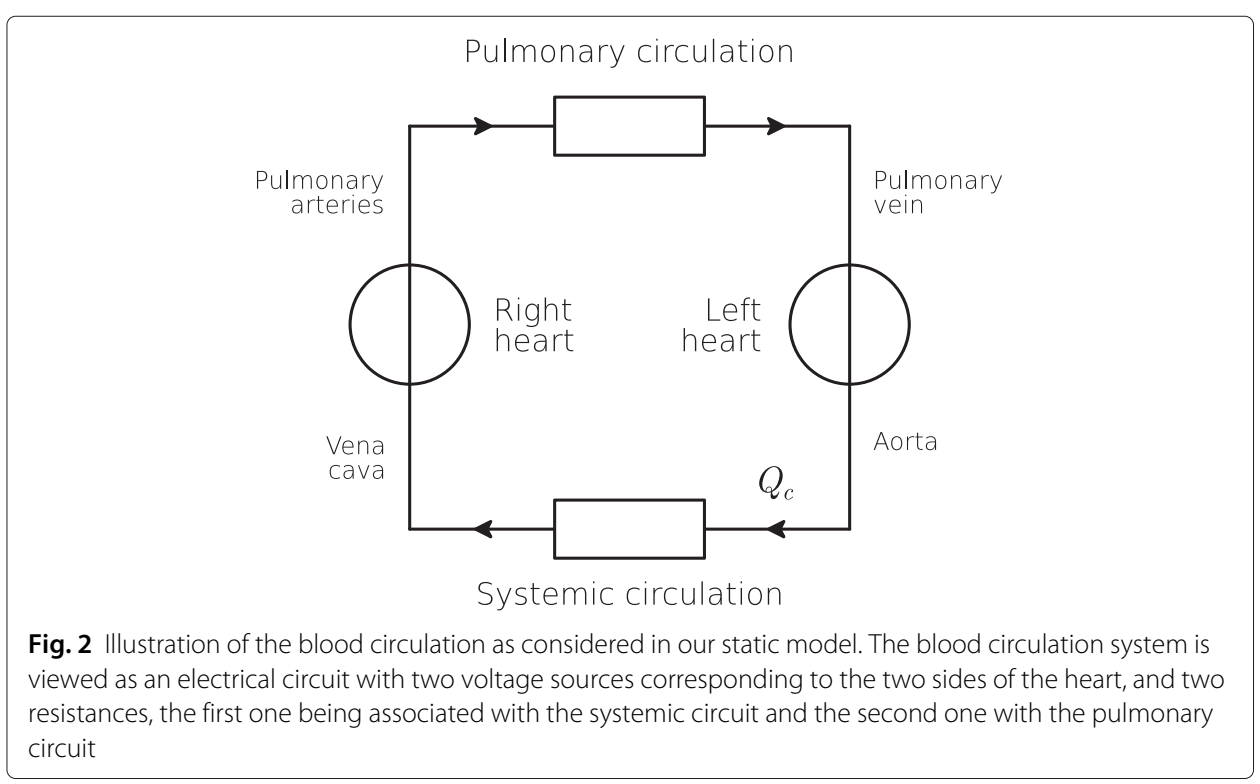


We supposed that the vena cava and pulmonary vein pressures are equal to the right and left atrial pressures, respectively. The powers delivered by the heart to ensure the cardiac output in the systemic and pulmonary circuits are given by

$$
\left\{\begin{array}{l}
\mathcal{P}_{\mathrm{r}}=\Delta P_{\mathrm{r}} \cdot Q_{c} \\
\mathcal{P}_{\mathrm{l}}=\Delta P_{\mathrm{l}} \cdot Q_{c}
\end{array}\right.
$$

where $\Delta P_{1 / \mathrm{r}}$ is the gradient of pressure between the input and the output of the left/right sides of the heart, respectively. For the left side of the heart, we have

$$
\mathcal{P}_{\mathrm{l}}=\left(P_{\mathrm{aa}}-P_{\mathrm{pv}}\right) \cdot Q_{c}=0.967 \mathrm{~W} .
$$

The power delivered by the right side of the heart is

$$
\mathcal{P}_{\mathrm{r}}=\left(P_{\mathrm{pa}}-P_{\mathrm{vc}}\right) \cdot Q_{c}=0.0956 \mathrm{~W},
$$

which is thus approximately equal to $10 \%$ of the power delivered by the left side of the heart. From this point of view the right side of the heart is rather weak compared to the left side.

\section{Sustained exercise situation}

To have a better perception of the ratio between the powers delivered by the right and left sides of the heart, we considered a well trained athlete. The values thus obtained will serve as references for discussing what may occur in the case of a pulmonary arterial hypertension. Let us suppose that, at exercise, athlete's heart can produce a cardiac output $Q_{c}$ up to $25 \mathrm{l} / \mathrm{min}$ [19] and an average aortic pressure up to $200 \mathrm{mmHg}$ [20]. We considered different exercise levels with the terminology suggested by BROWN et al. [19]. We estimated the aortic pressure at different exercise levels by a linear interpolation as suggested by these authors. The pulmonary arterial pressure thus depends on the cardiac output $Q_{c}$ according to

$$
P_{\mathrm{pa}}=0.94 Q_{c}+6.9
$$

which was obtained from the data provided in [21] and where the pressure is given in $\mathrm{mmHg}$ and the cardiac output in $1 / \mathrm{min}$. These data allow to compute the powers developed by the right and left sides of the heart during exercise (Table 1).

\section{Pulmonary arterial hypertension}

We now consider a patient suffering from a light pulmonary arterial hypertension, that is, from a pulmonary arterial hypertension compensated by a hypertrophy of the right side of

\begin{tabular}{|c|c|c|c|c|c|}
\hline $\begin{array}{l}\text { Exercise } \\
\text { level }\end{array}$ & $\begin{array}{l}Q_{C} \\
(1 / \min )\end{array}$ & $\begin{array}{l}P_{\text {aa }} \\
(\mathrm{mmHg})\end{array}$ & $\begin{array}{l}P_{\mathrm{pa}} \\
(\mathrm{mmHg})\end{array}$ & $\begin{array}{l}\mathcal{P}_{\mathrm{l}} \\
(\mathrm{W})\end{array}$ & $\begin{array}{l}\mathcal{P}_{\mathrm{r}} \\
(\mathrm{W})\end{array}$ \\
\hline Maximal & 25 & 200 & 30.4 & 10.67 & 1.522 \\
\hline Heavy & 17 & 157 & 22.9 & 5.63 & 0.752 \\
\hline Light & 9 & 115 & 13.4 & 2.14 & 0.208 \\
\hline Rest & 5 & 95 & 11.6 & 0.967 & 0.0956 \\
\hline
\end{tabular}
the heart which still ensures the nominal cardiac output. The pulmonary arterial pressure is set to $P_{\mathrm{pa}}=25 \mathrm{mmHg}$. The delivered power by the right ventricle at rest $\left(Q_{c}=5 \mathrm{l} / \mathrm{min}\right)$

Table 1 Hemodynamic characteristics for different exercise levels

Data are linearly interpolated between rest and maximal exercise values of a hobbyist athlete. Pulmonary arterial pressure is obtained using the linear regression (4) 
is thus

$$
\mathcal{P}_{\mathrm{r}}=\left(P_{\mathrm{pa}}-P_{\mathrm{vc}}\right) \cdot Q_{c}=0.244 \mathrm{~W},
$$

that is, the power provided during a light exercise (see Table 1 for comparison). There is no significant symptom.

Let us now consider a patient presenting a pulmonary arterial hypertension associated with a right ventricular failure (Fig. 1c). In this case, we considered a pressure $P_{\mathrm{pa}}^{\prime}$ equal to $50 \mathrm{mmHg}$. The power delivered by the right side of the heart to ensure the cardiac output at rest is now

$$
\mathcal{P}_{\mathrm{r}}^{\prime}=\left(P_{\mathrm{pa}}^{\prime}-P_{\mathrm{vc}}\right) \cdot Q_{c}=0.522 \mathrm{~W} .
$$

It corresponds to a power greater than twice $(251 \%)$ the power delivered during a light exercise. Moreover, contrary to an exercise for which the power increase is only delivered for a short duration, the cardiac power is here continuously delivered as for a patient with a pulmonary arterial hypertension: this could be a first explanation for the breathlessness symptom commonly associated with this pathology. Breathlessness here refers to the fact that the right ventricle is working at its maximum capacity thus inducing a poor oxygenation of the blood. The body would search for a more optimal solution for spending its energy since, in decompensated patients with a pulmonary arterial hypertension, the work required to ensure the cardiac output cannot be exclusively delivered by the right side of the heart. The power increase $(0.426 \mathrm{~W})$ that the right side of the heart has many difficulties to deliver could be very easily supplied by the left side of the heart, at least from the power point of view. Consequently, during the decompensation phase, the right ventricle fails to eject sufficient blood causing congestion and hence an increase in pressures. Contrary to what happens during the compensation phase, the power that the right ventricle is no longer able to deliver would be provided by the left ventricle; the right ventricle would thus work as it would do in a normal situation. In that case, to be able to deliver the required power in the pulmonary circuit, the left side of the heart would increase the mean pressure in the whole systemic circuit by $38.4 \mathrm{mmHg}$, increasing the vena cava pressure up to $P_{\mathrm{vc}}^{\prime}=41.4 \mathrm{mmHg}$ and inducing an increase of the aortic pressure to $P_{\mathrm{aa}}^{\prime}=133.4 \mathrm{mmHg}$. The power developed by the right ventricle would be therefore

$$
\mathcal{P}_{\mathrm{r}}=\left(P_{\mathrm{pa}}^{\prime}-P_{\mathrm{vc}}^{\prime}\right) \cdot Q_{c}=0.0956 \mathrm{~W},
$$

that is, the same work as produced at rest. The left side of the heart ensures henceforth the power

$$
\mathcal{P}_{\mathrm{l}}=\left(P_{\mathrm{aa}}^{\prime}-P_{\mathrm{pv}}\right) \cdot Q_{c}=1.39 \mathrm{~W},
$$

that is, a power still significantly less $(65 \%)$ than the one produced during a light exercise. According to our assumption, the heart has a great energetic advantage (from the working condition point of view) to ensure the cardiac output by the left side of the heart rather than by the right side.

This relay by the left heart has a side effect since the right side of the heart is overpressurized by $38.4 \mathrm{mmHg}$. Using the compliance of the right ventricle

$$
C=\frac{\mathrm{d} V}{\mathrm{~d} P}=4 \mathrm{~cm}^{3} / \mathrm{mmHg}
$$

as provided in [22], an increase of the pressure by $38.4 \mathrm{mmHg}$ in the right side of the heart implies an increase of its volume by $153.6 \mathrm{ml}$, that is, its volume roughly doubles, a 
result which is in agreement with the right ventricle dimensions observed in patient with a pulmonary arterial hypertension [23].

This simple static model does not allow to determine more exactly the respective contributions of the two sides of the heart for delivering the cardiac output. Nevertheless, it evidences that the left side of the heart could assist in a certain way the right side and allows to explain why the right side of the heart inflates during the decompensation phase. A more precise understanding of such assistance should be obtained with a dynamical model describing the cardiovascular system, taking into account a description of the main regulation mechanisms involved in chronic cardiac failure. It will therefore be possible to follow the evolution of the heart function when the pulmonary resistance is varied from a value corresponding to an healthy subject to a value associated with a patient in a decompensation state.

\section{A dynamical model}

Our aim is now to develop a dynamical model to understand the progression of the heart function during the development of a pulmonary arterial hypertension. One of the cardiovascular system functions is to control blood gas and the concentration of diverse elements (nutrient, waste, etc.) carried by blood. Practically, its actions are rather limited: the heart can only vary the cardiac output and the pressures; it does not have a direct influence on other factors. Consequently we focused our model on the control of the delivered cardiac output $Q_{\mathrm{cd}}$ onto a required cardiac output $Q_{\mathrm{cr}}$, ignoring complex underlying mechanisms.

Moreover, pressure and cardiac output variations during the cardiac cycle are not considered: for each cardiac cycle, only average values are taken into account. Since our model does not consider events shorter than a heart beat, capacitive and inductive effects due to blood inertia and vessel elasticity are neglected: we thus considered that the pulmonary and systemic circuits are only resistive. Since our aim is to reproduce the cardiac remodeling due to a long-term disease, the vasoconstriction and vasodilation were also neglected and, consequently, the pulmonary and systemic resistances only change at a low rate. Atria are not described in our model which therefore only considers a two-cavity heart, meaning that atria only play the role of buffer tanks when the ventricular pressure is small enough to allow the blood release in ventricular cavities during ventricular filling. Since the pulmonary and systemic circuits are only resistive, the blood is fully ejected from a ventricle to the other, justifying why there is no need to consider the atrial contraction during the ventricular filling.

\section{Control and evolution of the heart rate}

A first way to adjust the cardiac output is to change the cardiac frequency $f_{c}$ (or the heart rate) since

$$
Q_{\mathrm{cd}}=V_{\mathrm{es}} f_{c}
$$

where $V_{\text {es }}$ is the stroke volume. This is the single variable that can quickly change for delivering the cardiac output $Q_{\mathrm{cd}}$ at the required value $Q_{\mathrm{cr}}$. This regulation is a function of the difference between the required cardiac output $Q_{\mathrm{cr}}$ and the delivered one $Q_{\mathrm{cd}}$. The regulation of the heart rate acts as a feedback control of the cardiac output depending on 
the "error"

$$
\varepsilon=Q_{\mathrm{cr} n}-Q_{\mathrm{cd} n} .
$$

When the delivered cardiac output is less than the required cardiac output - as encountered in the beginning of a physical effort, for example - the heart rate increases until the error $\varepsilon$ becomes null. Contrary to this, when the delivered cardiac output is greater than the required cardiac output, the heart rate decreases. The evolution of the heart rate can thus be written as

$$
f_{c_{n+1}}=f_{c_{n}}+\frac{\beta}{f_{c_{n}}} \varepsilon h_{f}\left(f_{c_{n}}\right)
$$

where $f_{c_{n+1}}$ and $f_{c_{n}}$ are the heart rate at the $(n+1)$ th and $n$th cardiac cycle, respectively. $\beta$ is the increase rate of the heart rate and

$$
h_{f}\left(f_{c_{n}}\right)=\mid \begin{array}{ll}
\frac{f_{c_{n}}}{\beta \varepsilon}\left(\overline{f_{c}}-f_{c_{n}}\right) & \text { if } f_{c_{n}}+\frac{\beta}{f_{c_{n}}} \varepsilon \geq \overline{f_{c}} \\
\frac{f_{c_{n}}}{\beta \varepsilon}\left(f_{\underline{c}}-f_{c_{n}}\right) & \text { if } f_{c_{n}}+\frac{\beta}{f_{c_{n}}} \varepsilon \leq \underline{f_{c}} \\
1 & \text { otherwise }
\end{array}
$$

is an activation function expressing that the heart rate is bounded between a minimal frequency $f_{c}$ and a maximal frequency $\overline{f_{c}}$. According to the last notation, the cardiac output at the $n$th cycle is thus

$$
Q_{\mathrm{cd}_{n}}=V_{\mathrm{el} n} f_{c_{n}}
$$

where $V_{\mathrm{el} n}$ is the left ventricular stroke volume at the $n$th cycle.

\section{Evolution of the right and left end-diastolic volumes}

During a cardiac cycle, each ventricle ejects a defined quantity of blood called the stroke volume which is sent to the other ventricle via the pulmonary or the systemic circuits. The evolution of the end-diastolic volume of a given ventricle thus corresponds to the difference between the stroke volume ejected from a given ventricle and the stroke volume coming from the other ventricle. As a component of the systemic circuit, kidneys have the ability to adjust the blood volume ${ }^{1}$ by a volume $V_{\text {kid }}$ that they can produce or remove. Thus the right and left end-diastolic volumes $V_{\mathrm{dr} n+1}$ and $V_{\mathrm{dl} n+1}$ at the $(n+1)$ th cycle are given by

$$
\left\{\begin{array}{l}
V_{\mathrm{dr} n+1}=V_{\mathrm{dr} n}-V_{\mathrm{er} n}+V_{\mathrm{el} n}+V_{\mathrm{kid} n} \\
V_{\mathrm{dl} n+1}=V_{\mathrm{dl} n}-V_{\mathrm{el} n}+V_{\mathrm{er} n}
\end{array}\right.
$$

where $V_{\mathrm{dr} n}\left(V_{\mathrm{dl} n}\right)$ is the right (left) end-diastolic volume at the $n$th cardiac cycle, and $V_{\mathrm{er} n}\left(V_{\mathrm{el} n}\right)$ is the right (left) stroke volume at the $n$th cardiac cycle. We have thus a cycle-to-cycle map between the two end-diastolic volumes. We now have to express how the volume $V_{\mathrm{kid} n}$ produced by kidneys and the right (left) stroke volume $V_{\mathrm{er} n}\left(V_{\mathrm{el} n}\right)$ evolve.

\section{Control of the external volume}

A second way to adjust the cardiac output is to increase or decrease the total volume of blood in the cardiovascular system. As for the heart rate, we consider that the total blood volume is regulated by an error function between the required cardiac output $Q_{\mathrm{cr}}$ and the delivered one $Q_{\mathrm{cd}}$. If the delivered cardiac output $Q_{\mathrm{cd} n}$ is less than the required one $Q_{\mathrm{cr} n}$, kidneys increase the blood volume in the cardiovascular system. Contrary to 
this, if the delivered cardiac output is greater than the required cardiac output, some blood is drained from the cardiovascular system. The regulation of the blood volume $V_{\text {kid } n}$ (drained or injected) at the $n$th cardiac cycle is given by

$$
V_{\mathrm{kid} n}=\frac{\gamma}{f_{c_{n}}} \varepsilon+h_{V}\left(V_{\mathrm{dr} n}, V_{\mathrm{dl} n}\right)
$$

where $f_{c_{n}}$ is the heart rate at the $n$th cardiac cycle, and $\gamma$ is the volume that kidneys can produce or remove at each cycle. The $\gamma$-value is chosen in such a way that the evolution of the blood volume is slow compared to the variation of the cardiac frequency. Finally,

$$
h_{V}\left(f_{c_{n}}\right)=\mid \begin{array}{ll}
-\frac{\gamma}{f_{c_{n}}} \varepsilon & \text { if } \varepsilon>0 \text { and } V_{\mathrm{dr} n}+V_{\mathrm{dl} n} \geq \overline{V_{T}} \\
0 & \text { otherwise }
\end{array}
$$

is an activation function expressing the maximum total volume $\overline{V_{T}}$ that the heart can reach.

\section{Estimation of the right and left stroke volumes}

The cycle-to-cycle evolution of the heart rate (12) and the evolution of the end-diastolic volumes (15) depend on the right and left stroke volumes. In DE PATER and VAN DEN BERG like-models [11], the cavities are described using voltage generators producing a pressure corresponds to the contraction of the ventricles. Nevertheless, none of these models describes the muscular development due to the occurrence of a pathology like a pulmonary arterial hypertension.

The heart is a pump which can be viewed as a pressure generator producing the cardiac output, but we can also see it as a cardiac output generator producing the pressure. We prefer the latter point of view because our aim is to develop a mean model: we do not want to describe accurately the pressure or the cardiac output evolutions that are supposed to be constant during a cardiac cycle. Nevertheless we have information about the enddiastolic volume and the heart rate that easily leads to the cardiac output without knowing the pressure. Thus each ventricle is here described as a non-ideal cardiac output generator producing i) the ejected output $Q_{\mathrm{er}}\left(Q_{\mathrm{el}}\right)$ corresponding to the blood actually ejected from the right (left) ventricle and ii) some backflow outputs $Q_{r}\left(Q_{1}\right)$ corresponding to the blood retained in the ventricle (Fig. 3a).

Each of these two non-ideal current generators is made of an ideal current generator associated with a resistor - the so-called backflow resistance - in parallel. The ideal output generator ejects all the blood contained in the ventricle at the beginning of the contraction: this is the end-diastolic volume $V_{\mathrm{dr}}$ or $V_{\mathrm{dl}}$ (Fig. 3b). The backflow resistance is introduced to reproduce the blood volume retained in the ventricle: in other words, everything happens as if this resistance was driving the fraction of non-ejected blood. An increase of this resistance implies an increase of the contraction efficiency since less blood is retained in the ventricle. We supposed that an increase in the backflow resistance corresponds to the muscular development in the sense that this development leads to an increase in the heart efficiency. 


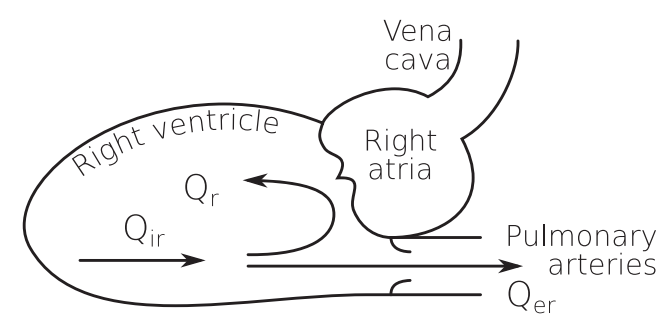

(a) Schematic diagram of the right ventricle ejection

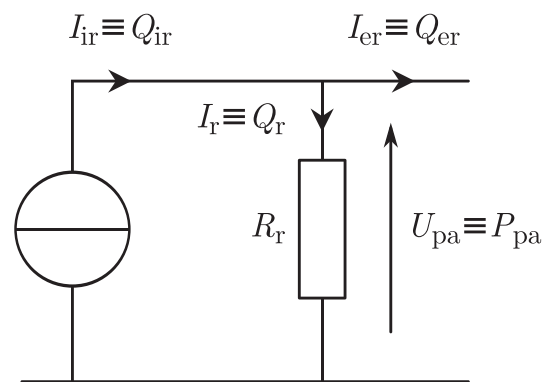

(b) Electrical analogy of the right ventricle during ejection

Fig. 3 Schematic diagram (a) of the right ventricle ejection and its electrical analogy $(\mathbf{b})$. The ventricle is considered as a non-ideal cardiac output generator made of an ideal generator providing the ideal output $Q_{\text {ir, }}$, then split into an ejected output $Q_{e r}$ corresponding to the blood actually ejected from the ventricle and a backflow output $Q_{r}$ corresponding to the blood retained in the ventricle. In the electrical analogy blood flow and pressure are replaced with current and voltage, respectively

To compute the stroke volume delivered by each ventricle, we used two electrical circuits corresponding to the pulmonary and the systemic circuits, respectively (Fig. 4). By definition, the right and left ideal cardiac outputs are

$$
\left\{\begin{array}{l}
Q_{\mathrm{ir}}=V_{\mathrm{dr}} f_{c} \\
Q_{\mathrm{il}}=V_{\mathrm{dl}} f_{c}
\end{array}\right.
$$

where $V_{\mathrm{dr}}$ and $V_{\mathrm{dl}}$ are the right and left end-diastolic volumes, and $f_{c}$ the heart rate. In a similar way, the ejected outputs are

$$
\left\{\begin{array}{l}
Q_{\mathrm{er}}=V_{\mathrm{er}} f_{c} \\
Q_{\mathrm{el}}=V_{\mathrm{el}} f_{c}
\end{array}\right.
$$

where $V_{\mathrm{er}}$ and $V_{\mathrm{el}}$ are the right and left stroke volumes, respectively. The right and left backflow outputs are defined according to

$$
\left\{\begin{array}{l}
Q_{\mathrm{r}}=\left(V_{\mathrm{dr}}-V_{\mathrm{er}}\right) f_{c}=V_{\mathrm{sr}} f_{c} \\
\mathrm{Q}_{\mathrm{l}}=\left(V_{\mathrm{dl}}-V_{\mathrm{el}}\right) f_{c}=V_{\mathrm{sl}} f_{c}
\end{array}\right.
$$

where $V_{\mathrm{sr}}$ and $V_{\mathrm{sl}}$ are the right and left end-systolic volumes, respectively. Let us designate by $R_{\mathrm{r}}$ and $R_{\mathrm{l}}$ the so-called backflow resistances in the right and left ventricles, respectively, and by $R_{\mathrm{p}}$ and $R_{\mathrm{s}}$ the pulmonary and systemic resistances. Using the definition of the backflow outputs and according to our electrical models Fig. 4), the pressure in the pulmonary artery and the aorta are

$$
\left\{\begin{array}{l}
P_{\mathrm{pa}}=R_{\mathrm{r}} Q_{\mathrm{r}}=R_{\mathrm{r}} V_{\mathrm{sr}} f_{c} \\
P_{\mathrm{aa}}=R_{\mathrm{l}} Q_{\mathrm{l}}=R_{\mathrm{l}} V_{\mathrm{sl}} f_{c},
\end{array}\right.
$$




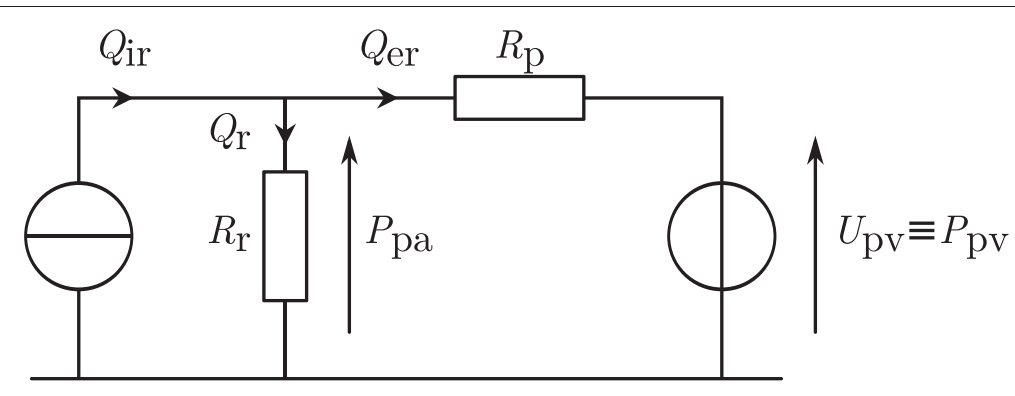

(a) Model of the pulmonary circuit

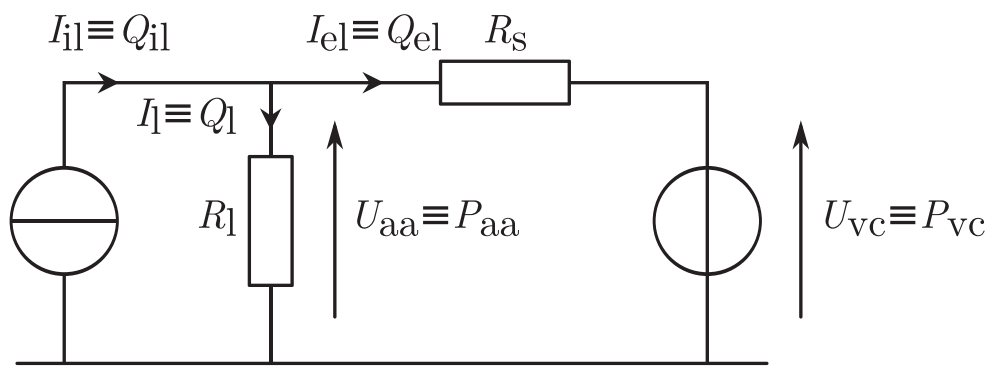

(b) Model of the systemic circuit

Fig. 4 Electrical models for the pulmonary (a) and systemic (b) circuits used for computing the stroke volumes delivered by the right and left ventricles, respectively

respectively. According to the electrical models (Fig. 4), the right and left ejected volumes at the $n$th cardiac cycle are

$$
\left\{\begin{array}{l}
V_{\mathrm{er} n}=V_{\mathrm{dr}_{n}} \frac{R_{\mathrm{r}_{n}}}{R_{\mathrm{r}_{n}}+R_{\mathrm{p}}}-\frac{1}{f_{c_{n}}} \frac{P_{\mathrm{pv}}}{R_{\mathrm{r}_{n}}+R_{\mathrm{p}}} \\
V_{\mathrm{el} n}=V_{\mathrm{dl}_{n}} \frac{R_{\mathrm{l}_{n}}}{R_{\mathrm{l}_{n}}+R_{\mathrm{s}}}-\frac{1}{f_{c_{n}}} \frac{P_{\mathrm{vc}}}{R_{\mathrm{l}_{n}}+R_{\mathrm{s}}} .
\end{array}\right.
$$

These relations between the end-diastolic volumes $\left(V_{\mathrm{dr}_{n}}\right.$ and $\left.V_{\mathrm{dl}_{n}}\right)$ and the stroke volumes ( $V_{\mathrm{er}_{n}}$ and $V_{\mathrm{el}_{n}}$ ) describe - with respect to the model structure - the Frank-Starling mechanism since an increase of the end-diastolic volume leads to an increase in the stroke volume. In real situation, there is a saturation of the stroke volume due to physiological limitation related to the decrease in the contraction efficiency when ventricles are over stretched. Although this effect is well known, there is, in the literature, no volume threshold at which the saturation occurs and the relationship between fiber length and ventricular volume is not analytically expressed. Consequently, we did not introduce any limiting factor to the Frank-Starling mechanism that is here supposed to be linear. Thus, using the right and left ejection fractions

$$
\rho_{\mathrm{r}_{n}}=\frac{V_{\mathrm{er} n}}{V_{\mathrm{dr} n}} \quad \text { and } \quad \rho_{\mathrm{l}_{n}}=\frac{V_{\mathrm{el} n}}{V_{\mathrm{dl} n}}
$$


which correspond to the proportions of blood actually ejected by the ventricles and defined as the ratio between the stroke volume $\left(V_{\mathrm{ex}}\right)$ and the end-diastolic volume $\left(V_{\mathrm{dx}}\right)$ of the considered ventricle $(x \in\{\mathrm{r}, \mathrm{l}\})$, the right and left backflow resistances are given by

$$
\left\{\begin{array}{l}
R_{\mathrm{r}_{n}}=\left(R_{\mathrm{p}}-\frac{P_{\mathrm{pv}}}{f_{c}}\right) \frac{\rho_{\mathrm{r}}}{1-\rho_{\mathrm{r}}} \\
R_{\mathrm{l}_{n}}=\left(R_{\mathrm{s}}-\frac{P_{\mathrm{vc}}}{f_{c}}\right) \frac{\rho_{\mathrm{l}}}{1-\rho_{\mathrm{l}}},
\end{array}\right.
$$

respectively. These backflow resistances could be thus estimated in clinics since all the parameters in Eq. (24) can be measured in patients.

\section{Estimation of the pulmonary vein and vena cava pressures}

The computation of the stroke volumes requires an estimation of the pressures $P_{\mathrm{pv}}$ in the pulmonary vein and $P_{\mathrm{vc}}$ in the vena cava that appear in Eq. (22). The left and right atrium pressures are supposed to be equal to these pressures. Since our model of the cardiovascular system has only two cavities, the volumes in the atria are not considered. Consequently, we need to approximate the venous pressures from the ventricles volumes. The blood ejected by one of the ventricle goes to the opposite atrium where it stays until the corresponding ventricle is in a relaxation state, during which the blood flows from the atrium to the ventricle. If for any reason the ventricle cannot eject a normal quantity of blood, the pressure inside the ventricle at the end of the ejection phase is greater than in a normal situation. Such a feature induces a decrease in the blood transfer from the atrium to the ventricle; the atrium pressure therefore increases too. It is thus possible to relate the vein pressure to the end-systolic volume of the ventricle. The pressures can be expressed as the products of a volume by an elastance (the opposite of a compliance), that is, according to

$$
\left\{\begin{array}{l}
P_{\mathrm{vc}}=E_{\mathrm{r}} V_{\mathrm{sr}} \\
P_{\mathrm{pv}}=E_{\mathrm{l}} V_{\mathrm{sl}},
\end{array}\right.
$$

where $P_{\mathrm{pv}}$ and $P_{\mathrm{vc}}$ are the pressures in the pulmonary vein and the vena cava, respectively; $E_{\mathrm{r}}\left(E_{\mathrm{l}}\right)$ is the right (left) ventricular elastance and $V_{\mathrm{sr}}\left(V_{\mathrm{sl}}\right)$ is the right (left) end-systolic volume.

In our model, we assumed that the elastance is not constant and can be agumented by an increase in the heart rate, in the wall thickness (represented by the $\bar{R}_{\mathrm{r}}$ and $\bar{R}_{\mathrm{l}}$ variables in this model) and in the volume of the ventricle cavity according to

$$
\left\{\begin{array}{l}
E_{\mathrm{r}}=\Gamma_{\mathrm{r}} f_{c} \bar{R}_{\mathrm{r}} V_{\mathrm{sr}} \\
E_{\mathrm{l}}=\Gamma_{\mathrm{l}} f_{c} \bar{R}_{\mathrm{l}} V_{\mathrm{sl}}
\end{array}\right.
$$

thus leading to

$$
\left\{\begin{array}{l}
P_{\mathrm{vc}}=\Gamma_{\mathrm{r}} f_{c_{n}} \bar{R}_{r n} V_{\mathrm{sr}_{n}}^{2} \\
P_{\mathrm{pv}_{n}}=\Gamma_{1} f_{c_{n}} \bar{R}_{l n} V_{\mathrm{sl}_{n}}^{2}
\end{array}\right.
$$

where $\Gamma_{\mathrm{r}}\left(\Gamma_{\mathrm{l}}\right)$ quantifies the dependence between i) the elastance and ii) the heart rate, the backflow resistance and the volume in the right (left) ventricle.

We supposed that an increase in the wall thickness leads to an increase in the wall stiffness and, consequently, to an increase in its elastance. When the heart rate increases, the 
ejection duration decreases more slowly than the duration of the filling phase. Consequently the volume in the atrium increases, leading to an increase in the veinous pressure: such a phenomenon can be viewed, in our model where atria are neglected, as an increase in the ventricular elastance. In a similar way, an increase in the end-systolic ventricular volume induces a decrease in the ventricular filling (more filled the ventricle is, more difficult to continue the filling is) and, consequently, induces an increase in the blood volume in the atrium: this effect is described as an increase in the elastance proportional to the volume which, as previously explained, should depend on the heart rate. To the best of our knowledge, the relationship between the ventricular volume and the veinous pressure was not studied and remains to validate. Such a dependency was important to be able to reproduce the proposed mechanism.

\section{Control and evolution of the right and left backflow resistances}

As we previously saw, increasing the backflow resistances can be used to model the muscular development. Describing their evolutions is thus equivalent to describe the evolution of myocardial cells. It is well-known that a subject at exercise develops his heart. Consequently, an athlete has thicker myocardial walls than a subject never practising sport. During strong exercise, the heart rate is strongly increased. A regular increase in the heart rate must lead to a muscular development of the heart, that is, according to our assumption, to an increase in the backflow resistances. Nevertheless, since our model is only developed for reproducing the long-term cardiac remodeling induced by chronic disease, exercises as performed by athletes are too short (typically one hour per day) for being correctly described. We would need to include some short-term regulation mechanisms as the baroreflex acting through pressure sensors in the aorta and carotid arteries, for instance.

Moreover, cardiac muscular fibers have an intrinsic mechano-sensing mechanism triggering their growth in response to an increase in load [24]. Such an increase in the cardiac mass normalizes the stress on the walls as a compensation mechanism. Nevertheless, if the volume increase is not bounded, the heart wall becomes too thick, inducing a lack of contractile function, that is, a heart failure [24]. Thus we supposed that an increase or a decrease in the end-diastolic volumes also lead to a change in the backflow resistances. In our model, cardiac remodeling is due to an increase in the heart and/or in the mechanical load: these two outputs are here treated in an independent way since there is no information concerning the way they could depend on each other.

Since an overload of the ventricle can damage myocardial cells [25], we rewrote the backflow resistance as

$$
R_{x}=\eta_{x} \bar{R}_{x} \quad(x \in\{\mathrm{r}, \mathrm{l}\})
$$

where $\eta_{x}$ is the contraction efficiency and $\bar{R}_{x}$ is the maximum backflow resistance. When there is a need for a muscular growth, although cardiac muscular fibers already reached their maximal size, the contraction efficiency slowly decreases from 1 to 0 .

Since the product of the heart rate by the end-diastolic volume has the unit of a flow rate and matches to the ideal cardiac output

$$
Q_{\mathrm{i} x}=f_{c_{n}} V_{\mathrm{d} x_{n}}
$$


of the considered ventricle, we supposed that the ventricle stabilizes its ideal cardiac output at the reference value $Q_{\mathrm{i} x}^{*}$. We therefore introduced the stabilization errors for the ideal cardiac outputs as

$$
\left\{\begin{array}{l}
\varepsilon_{\mathrm{r}_{n}}=f_{c_{n}} V_{\mathrm{dr}_{n}}-Q_{\mathrm{ir}}^{*} \\
\varepsilon_{\mathrm{l}_{n}}=f_{c_{n}} V_{\mathrm{dl}_{n}}-Q_{\mathrm{il}}^{*},
\end{array}\right.
$$

where $Q_{\mathrm{ir}}^{*}\left(Q_{\mathrm{il}}^{*}\right)$ designates the reference right (left) ideal cardiac output. In this approach, the ideal cardiac output is reached when the backflow resistance remains constant, that is, corresponding to a heart without muscular development. Contrary to this, a positive (negative) error $\varepsilon_{x}$ leads to an increase (decrease) in the backflow resistance representing a muscular development (atrophy).

Let us suppose that thicker the cardiac muscle, more difficult the muscular development becomes. This means that the increase rate of the maximal backflow resistance reaches the upper limit when this resistance is null and, is at its lowest limit when it goes to infinity. The cycle-to-cycle evolution of the maximum backflow resistances is thus as

$$
\bar{R}_{x n+1}=\bar{R}_{x_{n}}+\frac{\operatorname{sig}_{\lambda}\left(\varepsilon_{x n}\right)}{f_{c_{n}}} \mid \begin{array}{lll}
F_{x}\left(\bar{R}_{x_{n}}\right) & \text { if } \varepsilon_{x_{n}}>0 & \text { (development) } \\
\alpha_{x} & \text { otherwise } \quad \text { (atrophy) }
\end{array}
$$

where $\varepsilon_{x n}$ is the stabilization error of the ideal cardiac output given by Eq. (30) and, $x \in$ $\{r, l\}$ is a subscript for designating the right or left ventricle. When $\varepsilon_{x_{n}}<0$, the ideal cardiac output is too low, inducing an atrophy at the rate $\alpha_{x}$. Contrary to this, when the ideal cardiac output is too large $\left(\varepsilon_{x n}>0\right)$, a muscular development is induced, that is, the backflow resistance evolves according to

$$
F_{x}\left(\bar{R}_{x n}\right)=\bar{\alpha}_{x} f_{x}\left(\bar{R}_{x n}\right)
$$

where $\bar{\alpha}_{x}$ is the maximal increase rate. The function

$$
f_{x}\left(\bar{R}_{x n}\right)=\mid \begin{array}{ll}
\frac{R_{x}^{*}-\bar{R}_{x n}}{R_{x}^{*}} & \text { if } \bar{R}_{x n}<R_{x}^{*} \\
0 & \text { otherwise }
\end{array}
$$

represents the evolution of the increase rate of the backflow resistance where $R_{x}^{*}$ is the maximum value reached by the backflow resistance. We choose the $f_{x}$ function for producing a linear decrease from 1 to 0 zero when $\bar{R}_{x n}=R_{x}^{*}$ : this means that, in such a case, the increase in the ventricular muscle becomes more and more difficult, and finally stops when $\bar{R}_{\mathrm{x}_{n}}=R_{x}^{*}$. When the maximum value for the backflow resistance is reached, there is deleterous heart failure. Furthermore the function $\operatorname{sig}_{\lambda}$ in Eq. (31) has the form

$$
\operatorname{sig}_{\lambda}(x)=2\left(\frac{1}{1+e^{-\lambda x}}-\frac{1}{2}\right)
$$

where $\lambda$ defines the slope at the origin of the sigmoid function.

To complete our model, we designed the evolution of the contractility efficiency as

$$
\eta_{x_{n}+1}=\eta_{x_{n}}+\mid \begin{array}{ll}
-\frac{\sigma_{x}}{f_{c_{n}} \eta_{x_{n}}} & \text { if } f_{x}\left(R_{x_{n}}\right) \leq \underline{\varepsilon} \text { and } \varepsilon_{x_{n}}>0 \text { and } \eta_{x_{n}}>0 \\
0 & \text { otherwise }
\end{array}
$$

where $\sigma_{x}$ is the damage rate and $\underline{\varepsilon}$ is the damage threshold. This last equation establishes that when the maximum backflow resistance can no longer increase $\left(f_{x}\left(R_{x n}\right) \leq \underline{\varepsilon}\right)$ although an increase in this maximum backflow resistance $\left(\varepsilon_{x_{n}}>0\right)$ is expected, the contractility efficiency $\eta_{x_{n}}$ associated with this resistance decreases. 


\section{Synthesis}

Our model for heart remodeling can be synthesized as

$$
\left\{\begin{array}{l}
\bar{R}_{x n+1}=\bar{R}_{x_{n}}+\frac{\operatorname{sig}_{\lambda}\left(\varepsilon_{x_{n}}\right)}{f_{c_{n}}} \mid \begin{array}{l}
\bar{\alpha}_{x} f_{x}\left(\bar{R}_{x n}\right) \text { if } \varepsilon_{x_{n}}>0 \\
-\alpha_{x} \quad \text { otherwise }
\end{array} \\
\eta_{x_{n+1}}=\eta_{x_{n}}+\mid \begin{array}{c}
-\frac{\sigma_{x}}{f_{c_{n}} \eta_{x_{n}}} \\
\text { if } f_{x}\left(R_{x n}\right) \leq \underline{\varepsilon} \text { and } \\
\varepsilon_{x n}>0 \text { and } \eta_{x_{n}}>0 \\
\text { otherwise }
\end{array} \\
V_{\mathrm{dr} n+1}=V_{\mathrm{dr}_{n}}-V_{\mathrm{er} n}+V_{\mathrm{el} n}+V_{\mathrm{kid} n} \\
V_{c_{n+1}}=V_{\mathrm{dl} n}-V_{\mathrm{el} n}+V_{\mathrm{er} n} \\
=f_{c_{n}}+\frac{\beta}{f_{c_{n}}} \varepsilon_{n},
\end{array}\right.
$$

where

$$
\begin{aligned}
& \varepsilon_{x n}=f_{c_{n}} V_{\mathrm{d} x_{n}}-Q_{\mathrm{i} x}^{*} ; \\
& V_{\mathrm{kid} n}=\gamma \varepsilon_{n} ; \\
& \varepsilon_{n}=Q_{\mathrm{cr}}-Q_{\mathrm{cd} n} ; \\
& R_{x_{n}}=\eta_{x_{n}} \bar{R}_{x_{n}} ; \\
& f_{x}\left(\bar{R}_{x n}\right)=\mid \begin{array}{ll}
\frac{R_{x}^{*}-\bar{R}_{x_{n}}}{R_{x}^{*}} & \text { if } \bar{R}_{x n}<R_{x}^{*} \\
0 & \text { otherwise }
\end{array}
\end{aligned}
$$

with $x \in\{\mathrm{r}, \mathrm{l}\}$ and

$$
\left\{\begin{array}{l}
V_{\mathrm{er} n}=V_{\mathrm{dr} n} \frac{R_{\mathrm{r} n}}{R_{\mathrm{r} n}+R_{\mathrm{p}}}-\frac{\Gamma_{\mathrm{l}} f_{c_{n}} \bar{R}_{l n}\left(V_{\mathrm{dl} n-1}-V_{\mathrm{el} n-1}\right)^{2}}{R_{\mathrm{r} n}+R_{\mathrm{p}}} \\
V_{\mathrm{el} n}=V_{\mathrm{dl} n} \frac{R_{\mathrm{l} n}}{R_{\mathrm{l} n}+R_{\mathrm{s}}}-\frac{\Gamma_{\mathrm{r}} f_{c_{n}} \bar{R}_{r n}\left(V_{\mathrm{dr} n-1}-V_{\mathrm{er} n-1}\right)^{2}}{R_{\mathrm{l} n}+R_{\mathrm{s}}},
\end{array}\right.
$$

that were obtained by injecting Eq. (27) into Eq. (22).

\section{Pulmonary arterial hypertension modeling}

Our model was developed without any loss of generality, that is, it is not specific to any form of pathology and describes how a healthy subject can evolve under long-term mechanisms. More specifically, it was designed for studying the heart remodeling during the evolution of a chronic disease. In the case of a pulmonary arterial hypertension, the evolution of the pathology is simply reproduced by increasing the pulmonary resistance $R_{\mathrm{p}}$ versus time. This resistance is therefore no longer constant and becomes a variable of the model. The map for the pulmonary resistance is thus

$$
R_{\mathrm{p}_{n+1}}=R_{\mathrm{p}_{n}}+\frac{\Delta R_{\mathrm{p}}}{f_{c_{n}}}
$$


where $\Delta R_{\mathrm{p}}$ is the increase rate per beat of the pulmonary resistance. According to our model, an increase in the pulmonary resistance induces an increase in the pulmonary arterial pressure (Fig. 5).

Let us now investigate the case of a subject, initially healthy, who presents a slow increase in his pulmonary resistance $R_{\mathrm{p}}$ described by the map (43). Among the initial conditions reported in Table 2 and the parameter values in Tables 3 and 4, we choose a set of physiological values (for parameter marked with a "*”" in Tables 2, 3 and 4) corresponding to a healthy subject when we found them in the literature [19,21, 23, 26, 27]. The other parameter values were chosen to reproduce the decompensation phase in order to show the feasability of our approach. Our model does not use the cardiac output as a variable, but the right and left initial stroke volumes which were supposed to be equal to the initial values, that is,

$$
V_{\mathrm{er} 0}=V_{\mathrm{el} 0}=\frac{Q_{\mathrm{cr}}}{f_{c 0}}=70 \mathrm{ml}
$$

a value resulting from the initial cardiac frequency $f_{c 0}$ and the required cardiac output $Q_{\mathrm{ca}}$. These stroke volumes then allow to compute the initial values of the right and left maximum backflow resistances according to

$$
\left\{\begin{array}{l}
\bar{R}_{\mathrm{r} 0}=R_{\mathrm{p}} \frac{V_{\mathrm{er} 0}}{V_{\mathrm{dr} 0}-V_{\mathrm{er} 0}}+\frac{\Gamma_{\mathrm{l}} f_{c_{n}} \bar{R}_{\mathrm{l} 0}\left(V_{\mathrm{dl} 0}-V_{\mathrm{el} 0}\right)^{2}}{V_{\mathrm{dr} 0}-V_{\mathrm{er} 0}} \\
\bar{R}_{\mathrm{l} 0}=R_{\mathrm{s}} \frac{V_{\mathrm{el} 0}}{V_{\mathrm{dl} 0}-V_{\mathrm{el} 0}}+\frac{\Gamma_{\mathrm{r}} f_{c_{n}} \bar{R}_{\mathrm{r} 0}\left(V_{\mathrm{dr} 0}-V_{\mathrm{er} 0}\right)^{2}}{V_{\mathrm{dl} 0}-V_{\mathrm{el} 0}} .
\end{array}\right.
$$

Some of the parameter values used to characterize the left circuit (where the symbol " $\approx$ " is used) were based on the parameter values retained for the right circuit in such a way that

$$
\mu_{\mathrm{l}}=\frac{R_{\mathrm{l} 0}}{R_{\mathrm{r} 0}} \mu_{\mathrm{r}}
$$

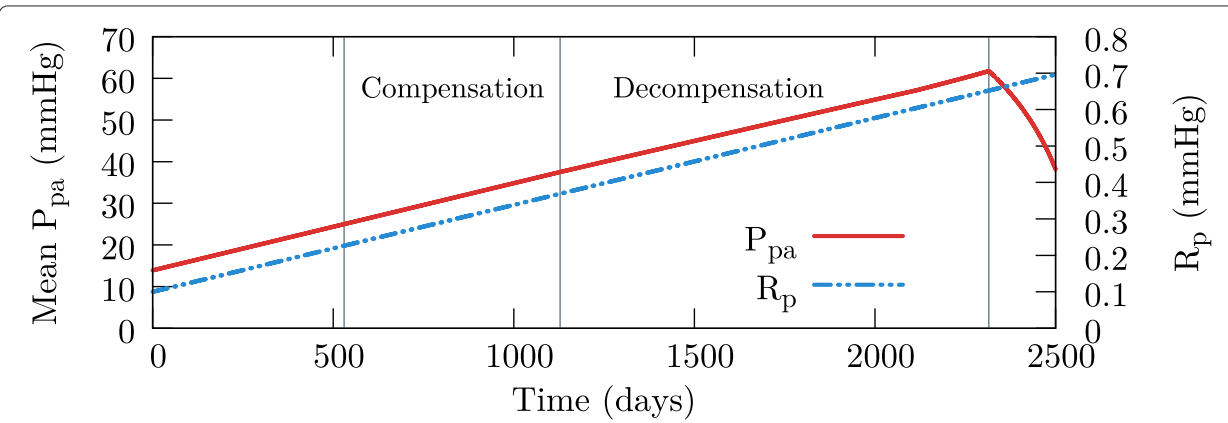

Fig. 5 Evolution of the mean pulmonary arterial pressure $P_{\mathrm{pa}}$ and the pulmonary resistance $R_{\mathrm{p}}$ versus time as described by our model for reproducing the evolution of a pulmonary hypertension. The graph can be divided into 4 parts: the first part (from day 0 to day 530) corresponds to the phase without any pulmonary arterial hypertension ( $P_{\text {pa }}<25 \mathrm{mmHg}$ ), the second part (from day 530 to day 1128 ) is the compensation phase, the third part (from day 1128 to day 2315) is the decompensation phase, and the last part (from day 2315) corresponds to the "death zone" during which a patient can not survive 
Table 2 Initial conditions used for our simulation

\begin{tabular}{lll}
\hline Right end-diastolic volume $^{*}$ & $V_{\mathrm{dro}}=$ & $120 \mathrm{ml}$ \\
Left end-diastolic volume $^{*}$ & $V_{\mathrm{dl0}}=$ & $135 \mathrm{ml}$ \\
Cardiac frequency $^{*}$ & $f_{c 0}=$ & $1.25 \mathrm{~Hz}(=75 \mathrm{bpm})$ \\
\hline Right backflow resistance & $\bar{R}_{\mathrm{r} 0} \approx$ & $0.222 \mathrm{mmHg} . \mathrm{s} / \mathrm{ml}$ \\
Left backflow resistance & $\bar{R}_{10} \approx$ & $1.08 \mathrm{mmHg} . \mathrm{s} / \mathrm{ml}$ \\
\hline
\end{tabular}

Parameters with a "**" were extracted from the literature

The initial flow $Q_{c d 0}=5.25 \mathrm{l} / \mathrm{min}$ is not an actual initial condition (because this is not a variable of our model) but is used for the computation of the right and left backflow resistances

where $\mu_{x}$ designates a given parameter value $(x \in\{\mathrm{r}, 1\})$ and $R_{\mathrm{r} 0}$ and $R_{\mathrm{l} 0}$ are the right and left backflow resistances at the initial time $(t=0)$. The ideal cardiac outputs were computed from the initial conditions (Table 2) using Eq. (20) leading to

$$
\left\{\begin{array}{l}
Q_{\mathrm{ir}}^{*}=f_{c_{0}} V_{\mathrm{dr} 0} \\
Q_{\mathrm{il}}^{*}=f_{c 0} V_{\mathrm{dl} 0} ;
\end{array}\right.
$$

these initial cardiac outputs are chosen to be stable, that is, associated with a null error, thus ensuring the stability of the initial conditions at $t=0$. We thus considered that the initial conditions correspond to a stable situation until $R_{\mathrm{p}}$ is varied. The evolution of the cardio-vascular dynamics is induced by the increase in the pulmonary resistance as observed in a pulmonary arterial hypertension syndrome. During our simulations, the subject was supposed to be at rest and, consequently, the required cardiac output $Q_{c r}$ remained constant.

\section{Results}

Our simulations (Fig. 6) reproduce the two-phase evolution of the disease. The first phase corresponds to the compensation during which only the right backflow resistance $R_{\mathrm{r}}$ increases, thus corresponding to a muscular development of the right ventricle (Fig. 6c). During the compensation phase, the other model variables remained at constant values (Fig. $6 \mathrm{a}$ and $6 \mathrm{~d}$ ). The increase in the right backflow resistance $R_{\mathrm{r}}$ is the main heart regulation mechanism ensuring the cardiac output in the pulmonary circuit while the pulmonary resistance $R_{\mathrm{p}}$ increases. Note that in our model, the increase in the pulmonary resistance $R_{\mathrm{p}}$ induces an

Table 3 Parameter values used in our model of the cardiovascular system with pulmonary arterial hypertension

\begin{tabular}{lrcc}
\hline & & General parameters & Units \\
\hline Required cardiac output * & $Q_{\mathrm{cr}}=$ & 87.5 & $\mathrm{ml} / \mathrm{s}$ \\
Slope of the sigmoid error function & $\lambda=$ & 100 & - \\
Increase rate of the cardiac frequency & $\beta=$ & $\frac{1}{60}$ & $\mathrm{~Hz} / \mathrm{ml}$ \\
Kidney volume exchange rate & $\gamma=$ & $2 \cdot 10^{-3}$ & $\mathrm{~s}$ \\
Minimal cardiac frequency & $\overline{f_{c}}=$ & $\frac{1}{60}$ & $\mathrm{~Hz}$ \\
Maximal cardiac frequency* & $\overline{f_{c}}=$ & $\frac{100}{60}$ & $\mathrm{~Hz}$ \\
\hline
\end{tabular}

Parameters with a "**" were extracted from the literature

This table contains general parameters (needed for the two parts of the heart) whereas parameter values specific to the left and right sides of the heart are reported in Table 4 . The required cardiac output chosen corresponds to $Q_{c r}=5.25 \mathrm{I} / \mathrm{min}$ 
Table 4 Parameter values used in our model of the cardiovascular system with pulmonary arterial hypertension

\begin{tabular}{|c|c|c|c|c|c|c|c|}
\hline \multirow[b]{2}{*}{ Development rate } & \multicolumn{3}{|c|}{ Left circuit } & \multicolumn{3}{|c|}{ Right circuit } & \multirow[b]{2}{*}{$\mathrm{mmHg} . \mathrm{s} / \mathrm{ml}$} \\
\hline & $\overline{\alpha_{l}}$ & $\approx$ & $9.75 \cdot 10^{-5}$ & $\bar{\alpha}_{\mathrm{r}}$ & $=$ & $2 \cdot 10^{-5}$ & \\
\hline Atrophy rate & $\alpha_{\uparrow}$ & $\approx$ & $3.9 \cdot 10^{-6}$ & $\alpha_{\mathrm{r}}$ & $=$ & $8 \cdot 10^{-7}$ & $\mathrm{mmHg} . \mathrm{s} / \mathrm{ml}$ \\
\hline Elastance coupling parameter & $\Gamma_{\mid}$ & $=$ & $9 \cdot 10^{-4}$ & $\Gamma_{\mathrm{r}}$ & $=$ & $9 \cdot 10^{-4}$ & $\mathrm{ml}^{-1}$ \\
\hline Backflow resistance limit & $R_{1}^{*}$ & $\approx$ & 2.93 & $R_{\mathrm{r}}^{*}$ & $=$ & 0.6 & $\mathrm{mmHg} . \mathrm{s} / \mathrm{ml}$ \\
\hline Circuit resistance * & $R_{\mathrm{s}}$ & $=$ & 1.0 & $R_{\mathrm{p}}$ & $=$ & 0.1 & $\mathrm{mmHg} . \mathrm{s} / \mathrm{ml}$ \\
\hline Ideal cardiac output * & $Q_{i}^{*}$ & $=$ & 168.75 & $Q_{i r}^{*}$ & $=$ & 150 & $\mathrm{ml} / \mathrm{s}$ \\
\hline Increase rate of pulmonary resistance & & & & $\Delta R_{\mathrm{p}}$ & $=$ & $2.76 \cdot 10^{-9}$ & $\mathrm{mmHg} / \mathrm{ml}$ \\
\hline Damage rate & $\sigma_{\mid}$ & $=$ & $4 \cdot 10^{-9}$ & $\sigma_{\mathrm{r}}$ & $=$ & $4 \cdot 10^{-9}$ & $\mathrm{~Hz}$ \\
\hline Damage threshold & $\underline{\varepsilon}_{\mid}$ & $=$ & $10^{-3}$ & $\underline{\varepsilon}_{\mathrm{r}}$ & $=$ & $10^{-3}$ & - \\
\hline
\end{tabular}

Parameters with a "**" were extracted from the literature

This table contains parameter values specific to the left and right sides of the heart whereas general parameters (needed for the two parts of the heart) are reported in Table 3. To simulate a healthy subject, $\Delta R_{p}=0$ would be used

increase in the pulmonary arterial pressure $P_{\mathrm{pa}}$ as a side effect: this variable is not involved in our model but can be straightforward obtained using the term $P_{\mathrm{pa}}$ in Eq. (21).

Since the resistance $R_{\mathrm{r}}$ of the pulmonary circuit increases as the disease progresses, the pressure $P_{\mathrm{pa}}$ in the pulmonary artery must increase to answer the required cardiac output $Q_{\mathrm{r}} \approx Q_{1}$ (which is the quantity that is kept constant in our model). Consequently, the power $\mathcal{P}_{\mathrm{r}}=P_{\mathrm{pa}} Q_{\mathrm{r}}=R_{\mathrm{r}} Q_{\mathrm{r}}^{2}$ must increase too. The right ventricle is able to compensate the evolution of the disease until the increase in the pulmonary resistance i) is slow enough to allow the muscular growth and, ii) does not require a too large increase in the power the right ventricle delivers. Indeed, when power $\mathcal{P}_{\mathrm{r}}$ becomes equivalent to those observed for a significant exercise, the subject becomes breathless due to the level of the right side of the heart activity. Such a compensation is working until the right side of the heart reaches its maximum development. Beyond this stage, the right side of the heart can no longer ensure the required cardiac output and an other mechanism to supply the blood circulation must be initiated. This is why the failure (or decompensation) phase occurs: since the first necessity is to ensure blood circulation, we here supposed that, during the decompensation phase, the left side of the heart starts to balance the right side failure by working against the pulmonary arterial resistance through the systemic circuit (Fig. 6c). In order to check the feasability of such a scenario, our model for long-term cardiac remodeling was thus proposed to be based on an increase in the heart rate and in a mechanical load. This model thus showed that for appropriate parameter values the right side of the heart is able to balance the increase in the pulmonary arterial resistance during the first phase of the disease (compensation) as shown in Fig. 6c. Then, the left side of the heart supplies the power to the blood flow that the right side of the heart can no longer provide (see Fig. 6).

Indeed, during the decompensation phase, the right side of the heart can no longer supply the required cardiac output since the right side reached its maximum value and its efficiency thus decreases (Fig. 7a), leading to a decrease in the backflow resistance (Fig. 6c). As a consequence of the decrease in the blood volume ejected from the right ventricle, there is thus a small blood volume which is carried from the left side to the right 


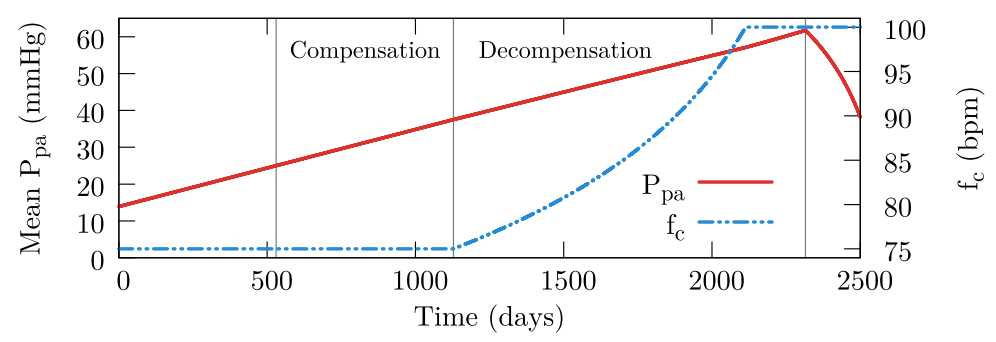

(a) Mean pulmonary arterial pressure $P_{\mathrm{pa}}$ and heart rate $f_{\mathrm{c}}$

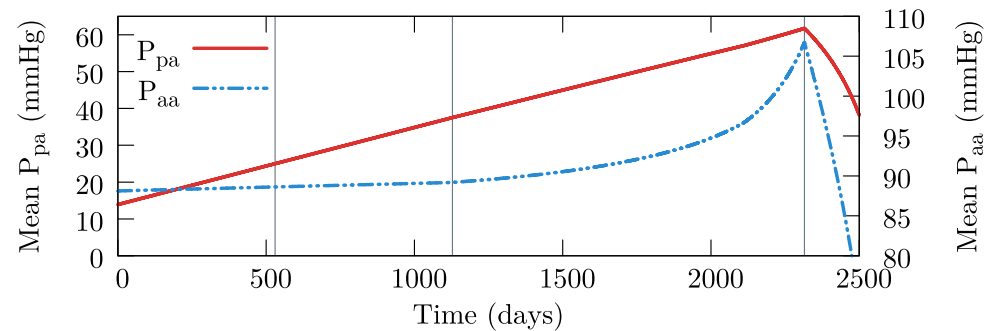

(b) Mean pulmonary arterial pressure $P_{\mathrm{pa}}$ and mean aortic pressure $P_{\text {aa }}$

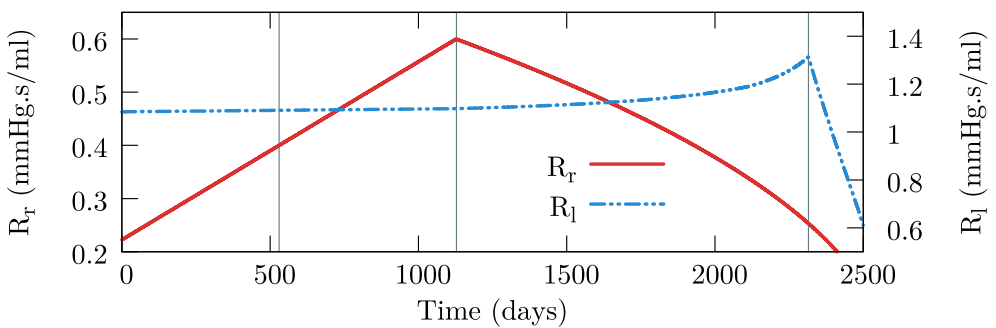

(c) Right and left backflow resistances

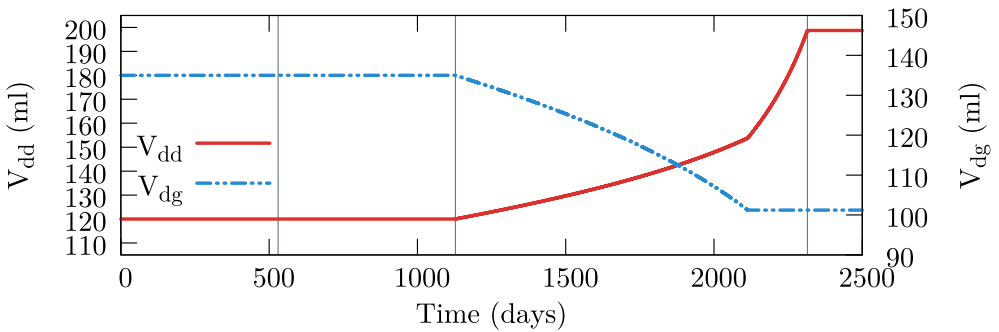

(d) Right and left end-diastolic volumes

Fig. 6 Evolutions of the different variables of our model when the mean pulmonary arterial pressure is progressively increased: mean pulmonary arterial pressure $(\mathbf{a}, \mathbf{b})$, heart rate $(\mathbf{a})$, mean aortic pressure $(\mathbf{b})$, right and left backflow resistances (c) and right and left end-diastolic volumes (d). The graph can be divided into 4 phases: the first phase (from day 0 to day 530 ) corresponds to the phase without any pulmonary arterial hypertension $\left(P_{\mathrm{pa}}<25 \mathrm{mmHg}\right.$ ), the second phase (from day 530 to day 1128 ) is the compensation phase, the third phase (from day 1128 to day 2315) is the decompensation phase. The last part (from day 2315) corresponds to the "death zone" during which the patient can no longer survive

side of the heart, but not from the right to the left side. Such a blood transfer is equal to about $2.16 \cdot 10^{-7} \mathrm{ml} \cdot \mathrm{min}^{-1}$ at the beginning of the decompensation phase and to about $3.99 \cdot 10^{-7} \mathrm{ml} \cdot \mathrm{min}^{-1}$ when the heart rate saturates at its maximal value (just before the increase in the volume due to the kidneys). The lack of blood in the left side of the heart induces a decrease in the cardiac output which is balanced by an increase in the cardiac 


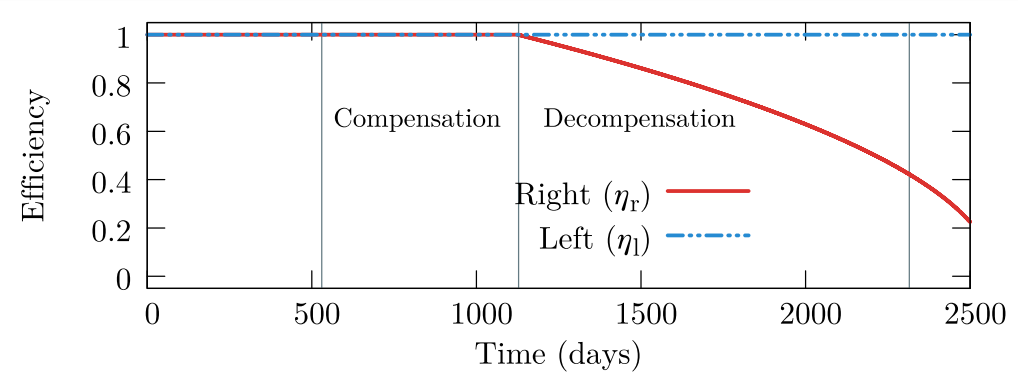

(a) Right and left efficiencies

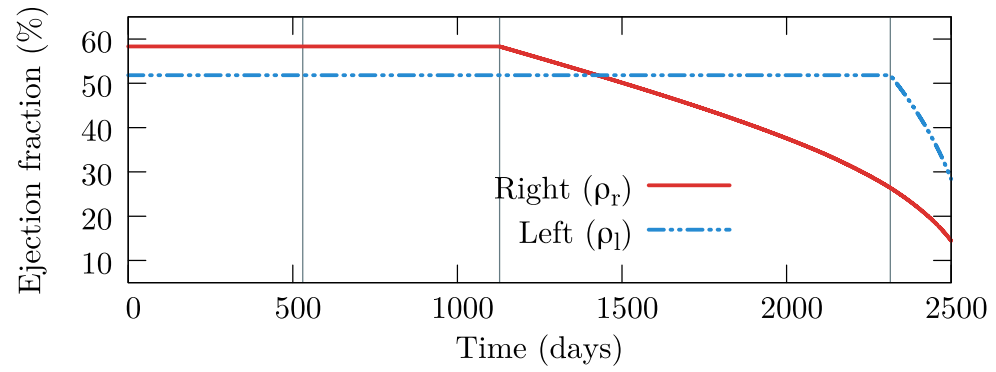

(b) Right and left ejection fractions

Fig. 7 Evolution of the efficiencies and the ejection fractions versus time. See caption of Fig. 6 for a description of the four phases

frequency for maintaining the blood flow in the systemic circuit at the required value. The increase in the heart rate thus induces an increase in the left backflow resistance, meaning a muscular development of the left side of the heart. The failure of the right side of the heart thus affects the left side. Moreover, a decrease in the ejection fraction of the right ventricle is observed when the ejection fraction of the left ventricle remains at a constant value (Fig. 7b), an effect sometimes clinically observed in a pulmonary arterial hypertension.

The increase in the pulmonary resistance leads to an increase in the power developed by the heart (Fig. 8). First, during the compensation phase, this increase is completely ensured by the right ventricle. When the right resistance to backflow saturates - and is therefore decreased - we observed an increase in the power delivered by the left ventricle: it shows that the left side assists the right one in extreme cases. We also observed that the increase in the power provided by the left ventricle is rather limited: in fact, we see that the true limiting factor is not the power but the saturation of all the regulation mechanisms. The main problem with a pulmonary arterial hypertension is that this is a disease whose evolution cannot be stopped, even with a treatment. Consequently, when the largest cardiac frequency authorized at rest in our model (100 bpm) and/or the largest mechanical load which can be supported by the heart is reached, the cardiac output decreases. These saturation phenomena lead to the death of the patient, here characterized by the decrease in almost all variables (Figs. 6 and 8) due to the decrease in the cardiac output: obviously, if the cardiac output is less than the required cardiac output, the whole cardiovascular status does not allow the survival of the patient. 


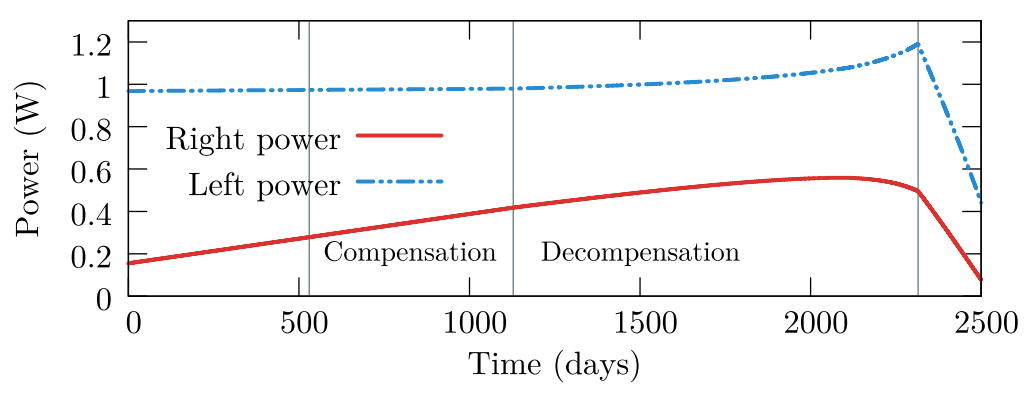

Fig. 8 Evolution of the powers delivered by the left and right ventricles

\section{Discussion}

This dynamical model of the cardiovascular system is an iterative model where each iteration corresponds to a new heart beat. This is a very interesting property of our model which should be further developed for taking into account short-term processes as those involved in the development of an athlete's heart, for instance.

As it is well known [5], an increase in the pulmonary resistance leads to two distinct phases. The first phase corresponds to a compensation during which the right ventricle muscle grows without clear clinical symptoms, the heart pumping efficiency being not affected. Such a compensation is followed by a cardiac failure state characterized by a decrease in heart performances as a decrease in the ejection fraction which is associated with an increase in the volume of the right cavity of the heart. There is not yet a clear explanation for these two phases. The one here proposed seems to be feasible, at least from the point of view of our model.

In the case of a pulmonary arterial hypertension, we performed a simulation for 2500 days (about 6 years and 10 months) of life (Figs. 6, 7 and 8); there is no need for a longer simulation since the last part is already non-physiological, that is, a patient could not survive when the cardiac output is no longer ensured.

Our model is not the first one to be designed for reproducing the cardiac remodeling as the one observed with a pulmonary arterial hypertension. Let us mention, for instance, the model developed by Rausch and co-workers [28] for describing the growth of soft biological tissue in the case of mechanically-induced wall thickening of the heart as observed in a moderate hypertension. Contrary to our model, this model does not explicitely take into account how the two ventricles can exchange blood and how the cardiac frequency is affected. As far as we understood, this model shows an increase in the volume of the right ventricle, even in the compensation phase, a fact which is not reported in the literature (see [29] for instance). Lumens and co-workers developed a multi-scale model coupling the natural mechanics via the interventricular septum to the hemodynamics dependency through the pulmonary and the systemic circulation [30]. They simulated different pathological situations, including a pulmonary arterial hypertension. Nevertheless, the transition between the two phases of this disease was not clearly shown as in our simulation. In particular, the inflation of the right ventricle only results from the stretched myocytes, which is in fact similar to what is observed with overloaded pressure [29]. Taking into account the appropriate regulation mechanisms and well-chosen parameter 
values, our model has therefore the advantage to show the possibility to explain the two phases clinically observed with a pulmonary arterial hypertension, just by increasing the pulmonary arterial resistance. Moreover, our model has also the advantage - compared to the two previous models - to work with a time step equal to the duration of one cardiac beat; short-term regulation mechanisms (other than the variation of the cardiac frequency) could be thus included in it. This is postponed for future works.

Even if the results obtained with this model seem to be very interesting, there are some limitations which should be mentioned. Due to the lack of acurate measurements in a cohort of patients, this study can only provide qualitative insights. In particular, some parts of this model would require a validation and perhaps some light modifications. One important limitation lies in the Frank-Starling mechanism, which is linearly modeled in our study. It means that an increase in the ventricular volume always leads to an increase in the cardiac output. We know that it is not rigorously true in particular when the volume takes large values, but we did not find threshold values beyond which this approximation is no longer valid. Consequently, to avoid to add two parameters with non justified values - one per side of the heart — we only considered a linear Frank-Starling mechanism.

Another important point consists in the dependency of the elastances of the pulmonary vein and of the vena cava on the heart rate and, in turns in the estimation of the venous pressure from the end-systolic volume, the heart rate and the resistance to backflow. The relationships we proposed influence all the variables since it has an effect on the stroke volume and on the power. Our results were very sensitive to the choice of that dependency and we tuned the parameter values to show the feasibility of the proposed mechanism. To the best of our knowledge, this type of relationship was not investigated in the literature and there is no available data to support (or not) our assumptions. We do believe that our results could open further studies in that directions.

Our model was clearly designed for reproducing long-term features as those induced by chronic disease like a pulmonary arterial hypertension. In order to keep it quite simple, we had to neglect some regulation loops as exemplified by the baroreflex acting through pressure sensors in the aorta and carotid arteries or by the beta adrenergic simulation inducing a modification of heart contractility. Consequently, the present model should be extended to reproduce some short-term features induced by daily exercises, for instance.

A last limitation of our model is related to the total volume growth. We supposed that the regulation of the total volume has a direct influence on the cardiac output, leading to an equation with the same form as the equation established for the heart rate. Due to an increase rate of the volume slower than those observed for the heart rate, the increase in the volume occurs after the saturation of the heart rate. Moreover, we did not model the variations of blood volume due to vasoconstriction and vasodilatation effects. To end this discussion, a not necessary trivial protocol should be designed to validate, at least partly, the hypothesis used for developing our model.

\section{Conclusion}

We first developed a static model to quickly check the feasability of a possible explanation for the inflation of the right ventricle during the decompensation phase of a pulmonary arterial hypertension. By using a mean behavior - meaning that the pulmonary and systemic circuits are only resistive - the model can predict the assistance of the right 
ventricle by the left one. The left ventricle increases the pressure in the systemic circuit to reduce the work produced by the right ventricle. This increase of pressure induces the inflation of the right ventricle. The resulting increase in the power delivered by the left ventricle only corresponds to a light exercise, while it was corresponding to a significant exercise when the right side of the heart was compensating the increased resistance of the pulmonary artery. There is thus an advantageous energetic compensation when the left side of the heart assists the right side of the heart.

In modeling the response of the heart to a pulmonary arterial hypertension by an increase in the backflow resistance, our dynamical model for the cardiovascular circulation was able to reproduce the two phases of this disease as they are clinically reported [5]. During the first phase, the right side of the heart balances the resistance increase by a muscular development (modeled by a backflow resistance), thus leading to an hypertrophied right side of the heart. The second phase corresponds to the failure of the right side of the heart to supply the cardiac output due to a large pulmonary resistance; the reduction of the cardiac output is balanced by a very slow blood transfer from the left to the right side of the heart, thus leading to an increase in the blood volume in the right ventricle, inducing an increase in the pressure in the inflated right side of the heart. As a consequence, the blood pressures in the aorta and in the vena cava are also increased. A pulmonary arterial hypertension is often associated with an elevated arterial pressure but when related to the left side of the heart, it is always considered that this is due to a "left heart disease" [31-33] and not to a possible compensation of the failure of the right side of the heart by its left side. The latter scenario, which is here proposed, is therefore not clinically documented. Our dynamical model also predicts an increase in the heart rate, a feature sometimes reported during decompensation.

Competing interests

The authors declare that they have no competing interests.

\section{Authors' contributions}

AK is the main contributor and author for the mathematical part of the dynamical model. CL is the main contributor and author for the mathematical part of the static model. BL and JM have contributed to the physiological and medical parts. All authors read and approved the final manuscript.

Author details

${ }^{1}$ CORIA UMR 6614 - Normandie University, CRNS-Université et INSA de Rouen, Campus Universitaire du Madrillet, F-76800 Saint-Etienne du Rouvray, France. ${ }^{2}$ ADIR Association, Avenue du Maréchal Juin, 76031 Rouen cedex, France.

${ }^{3}$ GRHV EA 3830, Universitary Hospital of Rouen, Hôpital de Bois-Guillaume, 76230 Bois-Guillaume, France.

Received: 19 May 2015 Accepted: 14 December 2015

Published online: 27 January 2016

References

1. Strumpher J, Jacobsohn E. Pulmonary hypertension and right ventricular dysfunction: Physiology and perioperative management. J Cardiothorac Vasc Anesth. 2011;25(4):687-704.

2. Montani D, Sitbon O, Fadel E, Datevelle P, Nunes H, Lebrec D, et al. Hypertension artérielle pulmonaire. EMC-Pneumologie. 2004;1:46-68.

3. von Romberg E. Über sklerose der lungenarterie. Deutsch Archiv für klinische Medizin. 1891;48:197-206.

4. Lourenço AP, Fontoura D, Henriques-Coelho T, Leite-Moreira AF. Current pathophysiological concepts and amangement of pulmonary hypertension. Int J Cardiol. 2012;155:350-61.

5. Champion HC, Michelakis ED, Hassoun PM. Comprehensive invasive and noninvasive approach to the right ventricle-pulmonary circulation unit : State of the art and clinical and reasearch implications. Circulation. 2009;120: 992-1007.

6. Nichols WW, O'Rourke MF. McDonald's Blood Flow in Arteries: Theoretical, Experimental and Clinical Principles. 3rd (ed), Edward Arnold. London; 1990

7. Hales S. Statistical Essays : Hæmastaticks vol. 2. Innys and Manby. Rome; 1733.

8. Weber EH. Comments on Anatomy and Physiology. C. F. Koehler: Leipzig; 1834 
9. Westerhof N, Lankhaar J-W, Westerhof BE. The arterial windkessel. Med Biol Eng Comput. 2009:47:131-141.

10. MK Sharp, WL Capper. Development of hydraulic model of the human systemic circulation. ASAIO J. 1999;45: 334-338.

11. De Pater L, Van Den Berg JW. An electrical analogue of the entire human circulatory system. Med Electron Biol Eng. 1964;2:161-166

12. Žácek M, Krause E. Numerical simulation of the blood flow in the human cardiovascular system. J Biomech. 1996;29:13-20.

13. Korakianitis T, Shi Y. A concentrated paramter model for the human cardiovascular system including heart valve dynamics and atrioventricular interaction. Med Eng Phys. 2006;28:613-28.

14. Fernandez de Canete J, del Saz-Orozco P, Moreno-Boza D, Duran-Venegas E. Object-oriented modeling and simulation of the closed loop cardiovascular system by using simscape. Comput Biol Med. 2013;43:323-333.

15. Smith BW, Andreassen S, Shaw GM, Jensen PL, Rees SE, Chase JG. Simulation of cardiovascular system diseases by including the autonomic nervous system into a minimal model. Comput Methods Programs Biomed. 2007;86: $153-60$.

16. Guyton AC, Coleman TG, Cowley AW, Liard J-F, Norman RA, Davis Manning R. Systems analysis of arterial pressure regulation and hypertension. Ann Biomed Eng. 1972;1:254-81.

17. Guyton AC, Jones CE, Coleman TG. Circulatory Physiology: Cardiac Output and Its Regulation. Saunders; 1973.

18. Sagawa K. Critique of a large-scale organ system model: Guytonian cardiovascular model. Ann Biomed Eng. 1975;3:386-400

19. Brown SP, Miller WC, Eason JM. Exercise Physiology. Lippincott Williams and Wilkins. Philadelphia; 2006.

20. Kurl S, Laukkanen JA, Rauramaa R, Lakka TA, Sivenius J, Salonen JT. Systolic blood pressure response to exercise stress test and risk of stroke. Stroke. 2001;32:2036-41.

21. Kovacs G, Berghold A, Scheid S, Olschewski H. Pulmonary arterial pressure during rest and exercise in healty subjects : a systematic review. Eur Respir J. 2009;34:888-94.

22. Matsumoto H. Measurement of instantaneous right ventricular compliance by phasic tricuspid flow velocity. Pediatr Cardiol. 1986;7:155-6.

23. Hilde JM, Skjørten I, Grøtta OJ, Hansteen V, Melsom MN, Hisdal J, et al. Right ventricular dysfunction and remodeling in chronic obstructive pulmonary disease without pulmonary hypertension. J Am Coll Cardiol. 2013;62:1103-11.

24. Bernardo BC, Weeks KL, Pretorius L, McMullen JR. Molecular distinction between physiological and pathological cardiac hypertrophy: Experimental findings and therapeutic strategies. Aliment Pharmacol Ther. 2010;128:191-227.

25. Pons-Lladó G, Ballester M, Borrás X, Carreras F, Carrió I, Lóprez-Contreras J, et al. Myocardial cell damage in human hypertension. J Am Coll Cardiol. 2000;36(7):2198-203.

26. Schlosser T, Pagonidis K, Herborn CU, Hunold P, Waltering K-U, Lauenstein TC, et al. Assessment of left ventricular parameters using 16-mdct and new software for endocardial and epicardial border delineation. Am J Roentgenol. 2005;184(3):765-73.

27. Kovalova S, Necas J, Vespalec J. What is a "normal" right ventricle?. Eur J Echocardiogr. 2006;7:293-7.

28. Rausch MK, Dam A, Göktepe S, Abilez OJ, Kuhl E. Computational modeling of growth Systemic and pulmonary hypertension in the heart. Biomech Model Mechanobiol. 2011;10(6):799-811.

29. Minami S, Onodera T, Okazaki F, Miyazaki H, Ohsawa S, Mochizuki S. Myocyte morphological characteristics differ between the phases of pulmonary hypertension-induced ventricular hypertrophy and failure. Int Heart J. 2006;47:629-37.

30. Lumens J, Delhaas T. Cardiovascular modeling in pulmonary arterial hypertension: Focus on mechanisms and treatment of right heart failure using the CircAdapt model. Am J Cardiol. 2012;1 10:39S-48S.

31. McLaughlin W, McGoon MD. Contemporary reviews in cardiovascular medicine: Pulmonary Arterial Hypertension. Circulation. 2006;114:1417-31.

32. Osenkranz S, Bonderman D, Buerke M, et al. Pulmonary hypertension due to left heart disease: updated recommendations of the Cologne Consensus Conference. Int J Cardiol. 2011;154:S34-S44.

33. Georgiopoulou W, Kalogeropoulos AP, Borlaug BA, Gheorghiade M, Butler J. Left ventricular dysfunction with Pulmonary Hypertension. Part 1, epidemiology, pathophysiology, and definitions, Circ Heart Fail. 2013;6:344-54.

\section{Submit your manuscript to a SpringerOpen ${ }^{\circ}$ journal and benefit from:}

- Convenient online submission

- Rigorous peer review

- Immediate publication on acceptance

- Open access: articles freely available online

- High visibility within the field

- Retaining the copyright to your article

Submit your next manuscript at $\boldsymbol{\triangleright}$ springeropen.com 